\title{
ASA classification: consistency of an universal tool.
}

\section{Pedrosa $\mathrm{E}^{1}$, Lobo A², Silva $\mathrm{MJ}^{3}$, Barbosa J2 , Mourão $\mathrm{J}^{1,2}$}

${ }^{1}$ Service of Anesthesiology, Hospital Center of São João, Porto, Portugal.

${ }^{2}$ Faculty of Medicine, University of Porto, Portugal.

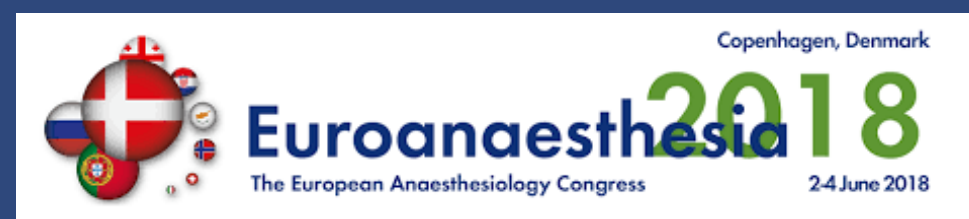

${ }^{3}$ Department of Anesthesiology, Portuguese Institute of Oncology, Porto, Portugal

\section{Introduction}

The classification of the physical state of the American Society of Anesthesiologists (ASA) (in 6 categories) is the most used classification in the preoperative evaluation by the anesthesiologists. Although it is related with anesthetic morbidity and mortality, inconsistent evaluations of the same patients among anesthesiologists have been reported. The aim of this study is to evaluate the variability of ASA physical status classification evaluation among Portuguese anesthesiologists.

\section{Methods}

Prospective study. An electronic questionnaire was distributed to the Portuguese anesthesiologists through the Portuguese Society of Anesthesiology mailing list and the social network Facebook for 7 days. Anesthesiologists were surveyed regarding their demographic characteristics, professional experience and workplace, and were asked to classify, according to ASA, the physical status of 15 clinical cases. The cases, similar to those of other studies, were translated by 2 anesthesiologists and 1 medical student. The agreement among participants was evaluated through intraclass correlation coefficient (ICC). A value of $p<0.05$ was assumed to be statistically significant.

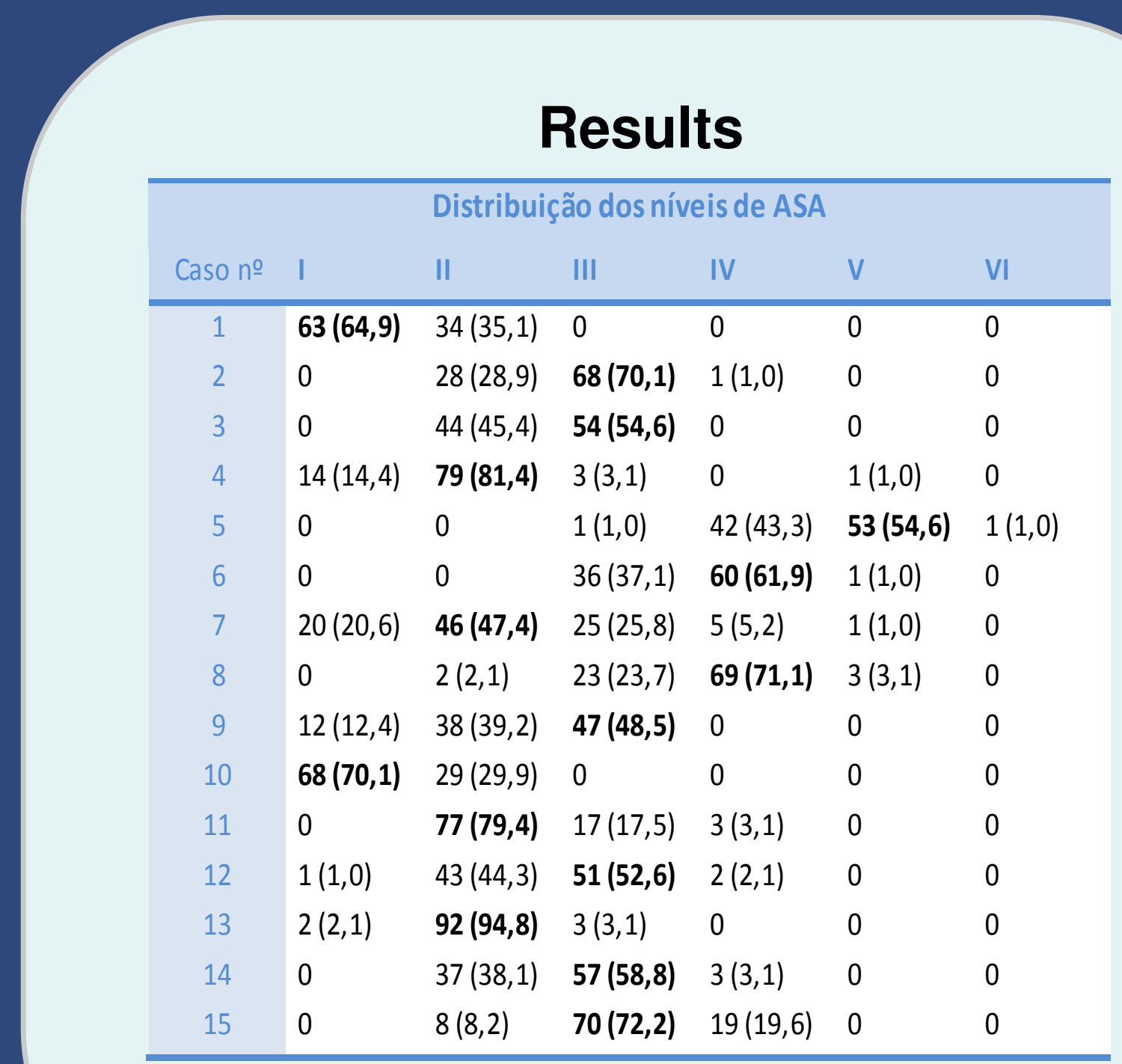

Table 1: 15 clinical cases ASA classification.
We obtained 100 responses, 3 were excluded due to lack of data. Mean age of participants: 38.76 were female and 21 male. 59 anesthesiology specialists and 38 specialty training interns. $93.8 \%$ work in the public sector. All participants assumed regular use of the ASA classification.

Compared with the original case classification, participants' agreement ranged from 3 to 15 responses, with a mean of $9.8(S D+/-2.3)$. In none of the cases was observed a complete agreement with the previous classification (max. 94.8\%). However, with the exception of 2 cases, in each clinical case the majority of participants had a concordant response. The ICC among the participants was $0.726(0.585,0.869, p<0.001)$ denoting a satisfactory degree of agreement (among the specialists, the value was 0.728 , and among the internal ones 0.709 ).

\section{Conclusion}

The results of this sample revealed that the agreement among Portuguese anesthetists is very satisfactory and is similar with the values observed in the literature. 